\title{
Analysis of Polarization-Maintaining 3-D Waveguide in Very Thin Multilayer Films Structure
}

\author{
Tetsuo Anada Hsu, Jui-Pang \\ Faculty of Engineering, Kanagawa University \\ 3-27 Rokkakubashi, Yokohama 221 JAPAN
}

Abstract- We propose and analyze a new optical three-dimensional waveguide having the function of a single-mode polarization-maintaining waveguide or TE/TM mode splitter.

The structure of a dielectric optical waveguide proposed here consists of very thin multilayer films of optical materials with two different refractive indices for the confinement of the height direction and the strong mode separation between TE and TM and the rib-type waveguide for the confinement of the lateral direction. The method of analysis is based on the "mode-matching method using the planar circuit equations" and a familiar microwave equivalent circuit for 3-D waveguide structures has been presented. This equivalent circuit derives the complete set of hybrid modes included mode conversion effects. In this paper, we also discuss on several fundamental properties of the guided mode for various structure parameters in the optical 3-D waveguide.

\section{Introduction}

Three dimensional planar-type dielectric waveguides are the building blocks for many fundamental devices in integrated optics, millimeter and sub-millimeter wave integrated circuits.

In particular, a single-mode and polarizationmaintaining planar-type three-dimensional (3-D) dielectric waveguides will be strongly needed in near future for the integration of optical circuits and other applications. For the conventional three or four layer rib-type waveguides, the propagating modes produce two families, namely the TE-and TM like modes. On the other hand, since very thin multilayer films of two isotropic materials with different refractive indices (e.g. $\mathrm{S}_{i} \mathrm{O}_{2}$ and $\mathrm{T}_{\mathrm{i}} \mathrm{O}_{2}$ ) exhibit the artificial birefringence properties[1-3], it is well-known that this structure arises a large mode-separation between the TE and TM modes. Therefore, it is expected that very thin multilayer films 3-D waveguide operates as the TE polarization-maintaing waveguide; only the TE modes propagate and TM modes are cut-off. Also, a multilayer consisting of high and low refractive index materials stratified alternately has the characteristic
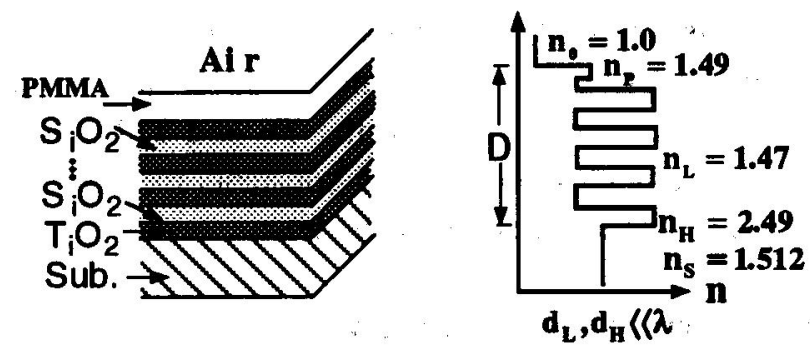

Fig. 1 Multilayer thin-film slab structure and refractive index profile.

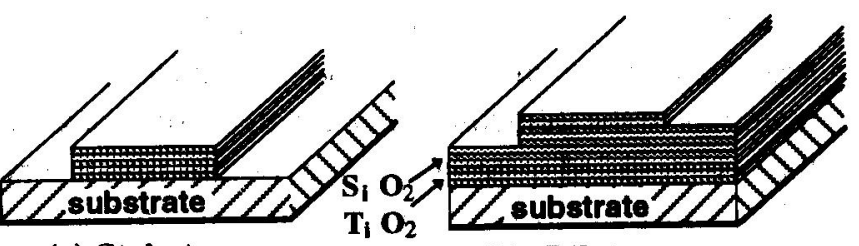

(a) Strip type

(b) Rib type

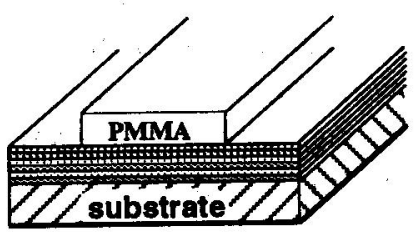

(c) Strip loaded type

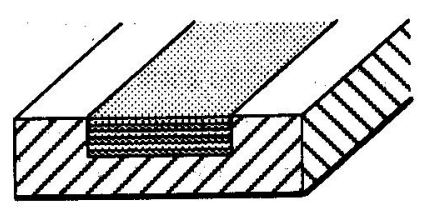

(d) Embedded type
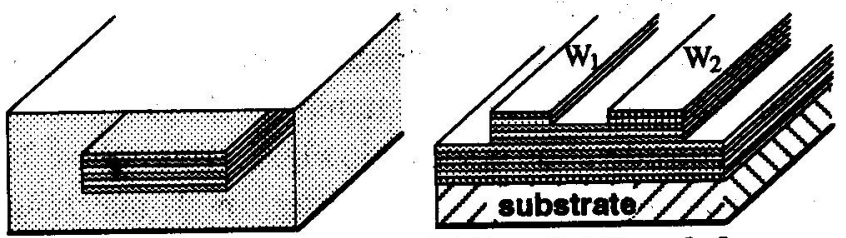

(d' ) Embedded type

(e) Parallel Coupled Waveguide

Fig. 2 Typical structure of the multilayer thin-film three dimensional optical waveguides.

$T_{1} O_{2}\left(n_{H}=2.49\right) S_{i} O_{2}\left(n_{L}=1.47\right)$ Substrate ( $\left.n_{S}=1.512\right)$

that an arbitrary refractive index value provides and this flexibility is desireable for integrated optics.

Several numerical or approximate methods for analyzing the multilayer thin-film structures have been developed to investigate the propagation characteristics of rib-type dielectric 3-D waveguides. Although the method described in [4]-[5] is quite useful for obtaining the propagation constant of 3-D waveguides, these approximate methods often break 
down for multilayer thin-films structures because of the abrupt discontinuities in refractive index. We propose a physically intuitive method for analyzing the multilayer thin-film 3-D waveguides; "the modematching method based on the planar circuit equations". Compared to other numerical techniques, this method described here has many advantages: first this method can obtain more accurate electromagnetic field distributions as well as the propagation constants of the guided modes. second,the nonphysical, spurious modes do not appear and third, it is easy to explain physical phenomena such as mode conversion effects between the TE and TM modes and the complex propagation constants for TM-like mode (in some cases, leaky mode).

\section{Structure and Working Mechanism}

The proposed multilayer structure consists of very thin-films stratified alternately with different dielectric materials as shown in Fig.1. This multilayer structure is used for the realization of a large separation between the TE and TM modes, where materials of the thin-films, the ratio of the thickness of the two stratified material, number of thin-film layers will contribute to the value of effective refractive index of the guided modes. The strip loaded-type or rib-type structures as shown in fig. 2 are also used for the mode suppression of the unwanted higher modes, where the thickness of strip will contribute to vary the effective refractive index. Through these mechanism, a single-mode and polarization maintaining 3-D waveguides are realized.

\section{Method of Analysis}

The typical rib-type structures of the multilayer thin-film polarization-maintaining 3-D waveguides under consideration is shown in Fig. 3. This optical 3$D$ waveguides are analyzed with the " mode-matching method" which utilizes the one-dimensional transmission-line matrix for the height (vertical) direction in multilayer thin-films and the transverse resonance relation based on the planar circuit equations for the horizontal direction [7]-[9]. Since the theoretical treatment has been described by authors, only the principle steps will be explained. The first step in the analysis is to find the height mode eigenfunction of waves propagating in multilayer thin-films slab for the structure parameters i.e., the total film thickness $\mathrm{D}(=\Sigma \mathrm{dH}+\Sigma \mathrm{dL})$, the ratio of each film thickness $R=(d H / d L)$, and number of layer $N$. At the rib-type sidewall discontinuity, an infinite number of TE and TM modes are excited, and are calculated for three slab-like regions (I, II and III) of the rib waveguide.
Using the appropriately defined mode voltage and mode vector current as shown in Table 1, then the total field components at each region can developed by a superposition of the vertical z-components (heighteigenmodes) of slab mode function in that region. That is

$$
\begin{aligned}
& E_{z}(x, y, z)=-\Sigma V_{n}^{E}(x, y) \cdot g_{n}^{E}(z) \\
& H_{z}(x, y, z)=-\Sigma V_{n}^{H}(x, y) \cdot g_{n}^{H}(z) \\
& E_{1}(x, y, z)=\Sigma\left[J_{p}^{H}(x, y) \times k\right] \cdot f_{0}^{H}(z) \\
& +j \eta_{0} \Sigma J_{\mathrm{n}}^{\mathrm{H}}(\mathrm{x}, \mathrm{y}) \cdot \mathrm{h}_{\mathrm{v}}^{\mathrm{H}}(\mathrm{z}) \\
& \mathbf{H}_{\mathrm{t}}(\mathrm{x}, \mathrm{y}, \mathrm{z})=\mathrm{j} \eta_{0}^{-1} \Sigma \mathbf{J}_{\mathrm{n}}^{\mathrm{H}}(\mathrm{x}, \mathrm{y}) \cdot \mathrm{h}_{\mathrm{n}}^{\mathrm{H}}(\mathrm{z}) \\
& +\Sigma\left[k \times J_{n}^{E}(x, y)\right] \cdot f_{n}^{E}(z)
\end{aligned}
$$

where $f(z), g(z), h(z)$ functions are defined by table 1

The second step is the evaluation of the lateral confinement for the rib-type waveguide having the structure parameters described above.

In these analysis, sufficient number of each TE and TM mode are taken into consideration in order to satisfy the continuity condition of the tangential field components at the step discontinuity in waveguide. The continuity of the transverse fields is translated in the continuity of mode voltages and currents at the interface plane.

Hence, the following equations are derived.

$$
\begin{aligned}
& \mathrm{E}_{\mathrm{z}}: \sum \mathrm{V}_{\mathrm{a}}^{1 \mathrm{E}} \cdot \mathrm{g}_{\mathrm{n}}^{\mathrm{IE}}(\mathrm{z})=\sum \mathrm{V}_{\mathrm{n}}^{2 \mathrm{E}} \cdot \mathrm{g}_{\mathrm{n}}^{2 \mathrm{E}}(\mathrm{z}) \\
& \mathrm{H}_{\mathrm{z}}: \Sigma \mathrm{V}_{\mathrm{n}}^{1 \mathrm{H}} \cdot \mathrm{g}_{\mathrm{n}}^{1 \mathrm{H}}(\mathrm{z})=\sum \mathrm{V}_{\mathrm{n}}^{2 \mathrm{H}} \cdot \mathrm{g}_{\mathrm{n}}^{2 \mathrm{H}}(\mathrm{z}) \\
& \mathrm{E}_{\mathrm{y}}:-\sum \mathrm{J}_{\mathrm{n} \perp}^{\mathrm{HH}} \cdot \mathrm{f}_{\mathrm{n}}^{1 \mathrm{H}}(\mathrm{z})+\mathrm{j} \eta_{0} \sum \mathrm{J}_{\mathrm{n} / 1}^{1 \mathrm{E}} \cdot \mathrm{h}_{\mathrm{n}}^{1 \mathrm{E}}(\mathrm{z})= \\
& \sum J_{n \perp}^{2 \mathrm{H}} \cdot \mathrm{f}_{\mathrm{n}}^{2 \mathrm{H}}(\mathrm{z})+\mathrm{j} \eta_{0} \sum \mathrm{J}_{\mathrm{n}}^{2 \mathrm{E}} \cdot \mathrm{h}_{\mathrm{n} / /}^{2 \mathrm{E}}(\mathrm{z}) \\
& \mathrm{H}_{\mathrm{y}}: \sum \mathrm{J}_{\mathrm{n} L}^{1 \mathrm{E}} \cdot \mathrm{f}_{\mathrm{n}}^{1 \mathrm{E}}(\mathrm{z})+\frac{1}{\mathrm{j} \eta_{0}} \sum \mathrm{J}_{\mathrm{n} / /}^{1 \mathrm{H}} \cdot \mathrm{h}_{\mathrm{n}}^{1 \mathrm{H}}(\mathrm{z})= \\
& -\sum \mathrm{J}_{\mathrm{nL}}^{2 \mathrm{E}} \cdot \mathrm{f}_{\mathrm{n}}^{2 \mathrm{E}}(\mathrm{z})+\frac{1}{\mathrm{j} \eta_{0}} \sum \mathrm{J}_{\mathrm{n} / /}^{2 \mathrm{H}} \cdot \mathrm{h}_{\mathrm{n}}^{2 \mathrm{H}}(\mathrm{z})
\end{aligned}
$$

where the order of $\mathbf{n}$ is the number of discretized continuum modes used in the field expansions in the inner and outer regions.

Applying the orthogonality relations shown in Table 1, mode coupling equations are derived.

At the discontinuity plane of rib-side wall, many TE and TM modes are generated to satisfy the boundary conditions. Enough number of height modes of each TE and TM mode then are taken into account according to the accuracy required. In this case, 25 TE and 25 TM modes are taken into consideration at each region, respectively. We leads to the desired mode coupling equations for the TE and TM modes and these relations are given as follows. 
$\left[\begin{array}{l}\mathbf{J}_{\perp}^{1 H} \\ \mathbf{J}_{\perp}^{1 \mathrm{E}}\end{array}\right]=\left[\begin{array}{ll}\mathbf{Y}_{\text {eff }}^{1 \mathrm{H} 1 \mathrm{H}} & \mathbf{Y}_{\mathrm{ef}}^{1 \mathrm{HIE}} \\ \mathbf{Y}_{\text {eff }}^{1 \mathrm{E}} & \mathbf{Y}_{\text {eff }}^{1 \mathrm{EIE}}\end{array}\right]\left[\begin{array}{l}\mathbf{V}^{1 \mathrm{H}} \\ \mathbf{V}^{1 \mathrm{E}}\end{array}\right]=\mathbf{Y}_{\mathbf{W}}^{(1)} \mathbf{V}^{(1)}$

$Y_{\text {eff }}^{1 \mathrm{H} 1 \mathrm{H}}=F^{1 \mathrm{H} 2 \mathrm{H}} \mathbf{Y}_{\mathrm{c \perp}}^{2 \mathrm{H}}\left(F^{1 \mathrm{H} 2 \mathrm{H}}\right)^{\mathrm{t}}$

$\mathbf{Y}_{\text {eff }}^{1 \mathrm{E} 1 \mathrm{E}}=\mathbf{F}^{1 \mathrm{E} 2 \mathrm{E}} \mathbf{Y}_{\mathrm{c \perp}}^{2 \mathrm{E}}\left(\mathbf{F}^{1 \mathrm{E} 2 \mathrm{E}}\right)^{\mathfrak{t}}$

$\mathbf{Y}_{\text {eff }}^{1 \mathrm{H} 1 \mathrm{E}}=j \eta_{0}\left\{\mathbf{H}^{1 \mathrm{HIE}} \mathbf{Y}_{\mathrm{c} / 1}^{\mathrm{IE}}-\mathbf{H}^{1 \mathrm{H} 2 \mathrm{E}} \mathbf{Y}_{c / 1}^{2 \mathrm{E}}\left(\mathbf{F}^{1 \mathrm{E} 2 \mathrm{E}}\right)^{\mathrm{t}}\right\}$

$Y_{\text {eff }}^{1 \mathrm{E} 1 \mathrm{H}}=\frac{1}{\mathrm{j} \eta_{0}}\left\{\mathbf{H}^{1 \mathrm{E} 1 \mathrm{H}} \mathbf{Y}_{\mathrm{c} / 1}^{1 \mathrm{H}}-\mathbf{H}^{1 \mathrm{E} 2 \mathrm{H}} \mathbf{Y}_{\mathrm{c} / 1}^{2 \mathrm{H}}\left(\mathbf{F}^{1 \mathrm{H} 2 \mathrm{H}}\right)^{\mathrm{t}}\right\}$

where mode coupling coefficients are defined as the overlap integrals of the height mode in the inner and outer regions.

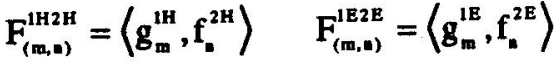

$$
\begin{aligned}
& H_{(m, 0)}^{1 H 1 E}=\left\langle g_{m}^{1 H}, h_{n}^{1 B}\right\rangle \quad H_{(m, n)}^{1 E 1 H}=\left\langle g_{m}^{1 E}, h_{n}^{1 H}\right\rangle \\
& H_{(m, n)}^{1 H 2 E}=\left\langle g_{m}^{1 H}, h_{a}^{2 E}\right\rangle \quad H_{(m, n)}^{1 E 2 H}=\left\langle g_{m}^{1 E}, h_{a}^{2 H}\right\rangle \\
& \left\langle g_{m}, f_{i}\right\rangle=\int g_{m}(z) f_{a}(z) d z
\end{aligned}
$$

Characteristics admittance are defined as follows.

$$
Y_{c \perp}=Y_{c} \frac{\beta_{x}}{\sqrt{\beta_{t}^{2}-\beta_{y}^{2}}} \quad Y_{c / l}=Y_{c} \frac{\beta_{y}}{\sqrt{\beta_{t}^{2}-\beta_{y}^{2}}}
$$

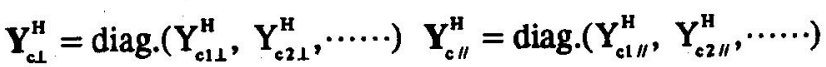

Similary, $Y_{w}^{(2)}$ (wall admittance looking into the left direction from inner region) is calculated in the same way.

With reference to Fig.3, the waveguide has two basic building blocks, i.e. uniform transmission line portions of multilayer thin-film slab guides and transformers which represents the step discontinuity. Since the equivalent circuit model for each constituent are obtained, the following equations are derived and by solving this eigenvalue equations, the propagation constant and field distributions of the waveguide mode can be given.

$$
\left[\begin{array}{c}
-\mathbf{Y}_{\mathrm{c} \perp} \cot \boldsymbol{\beta}_{\perp} \mathbf{W}+\mathbf{Y}_{\mathrm{W}}^{(1)} \\
\mathrm{j} \mathbf{Y}_{\mathrm{c} \perp} \operatorname{cosec} \boldsymbol{\beta}_{\perp} \mathbf{W} \\
\left.\quad \begin{array}{l}
\mathrm{j} \mathbf{Y}_{\mathrm{c} \perp} \operatorname{cosec} \boldsymbol{\beta}_{\perp} \mathbf{W} \\
-\mathbf{Y}_{\mathrm{c} \perp} \cot \boldsymbol{\beta}_{\perp} \mathbf{W}+\mathbf{Y}_{\mathrm{W}}^{(2)}
\end{array}\right]\left[\begin{array}{c}
\mathbf{V}^{(1)} \\
\mathbf{V}^{(2)}
\end{array}\right]=0
\end{array}\right.
$$

\section{Numerical Results}

We will describe several results for the multilayer thin-film polarization-maintaining 3-D waveguides as shown in Fig. 3. The structure parameters have been chosen to refractive index of $\mathrm{T}_{i} \mathrm{O}_{2}\left(n_{H}=2.49\right)$ and $\mathrm{S}_{\mathrm{i}} \mathrm{O}_{2}\left(\mathrm{n}_{\mathrm{I}}=1.47\right)$ materials, the ratio of thin-film layer $\mathrm{d}_{\mathrm{H}} / \mathrm{d}_{\mathrm{I}}=0.2$, the total thin-film thickness $\mathrm{D}=$ $0.6 \mu \mathrm{m}$, and the operating wavelength is assumed to be $\lambda=0.6328 \mu \mathrm{m}$.

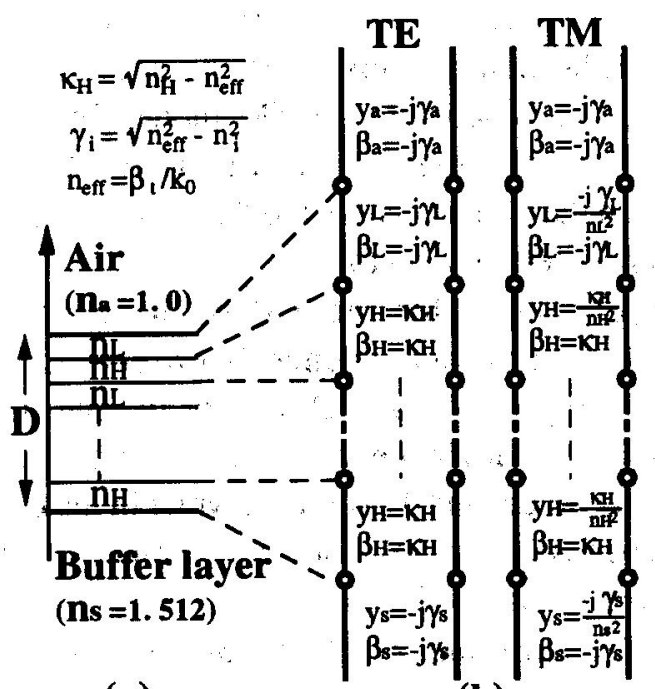

(a)

(b)

Height direction . Transmission line
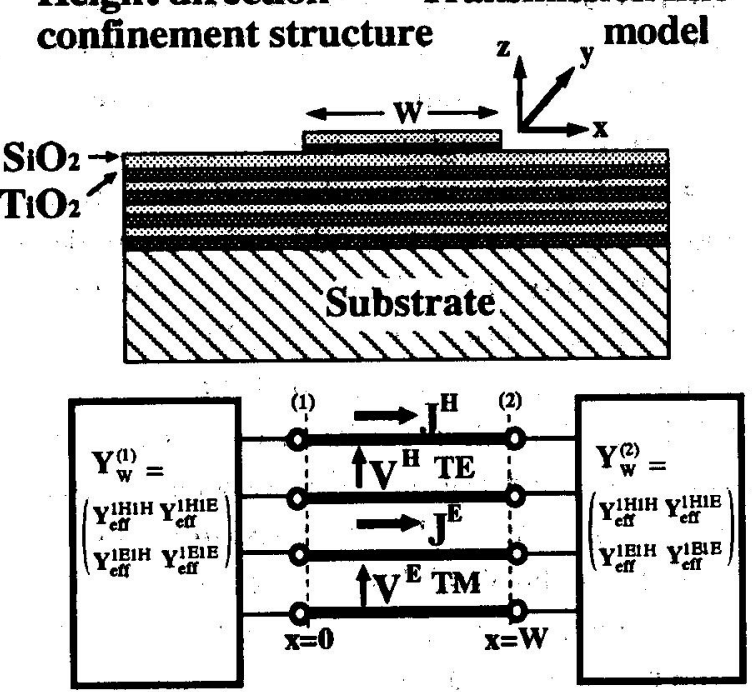

(b) Transverse equivalent circuit

Fig. 3 A rib waveguide and Its microwave equivalent circuit

\section{A. propagation properties of multilayer thin-film slab structures}

The numerical results are calculated for the multilayer thin-films slab. Fig.4 shows values of the effective index $n=\beta / k_{0}$ as a function of stratfied thin-film layer number $\mathrm{N}$ and the corresponding field distributions in height direction. Fig.4(b) shows the dispersion curves as a function of thickness of PMMA (dp) loaded on multilayer thin-films slab structure. As the thickness of PMMA increases from 0 up to $1.0 \mu \mathrm{m}$, the effective refractive index for the dominant mode increases from 1.655 to 1.658 value and is saturated around the thickness of $0.4 \mu \mathrm{m}$.

Therefore, in this range of the effective refractive index between PMMA thickness $d p=0$ and $d p=0.4$ $\mu \mathrm{m}$, there exists guided modes of PMMA loaded-type 3-D waveguide with multilayer thin-films ( $N=10$ layers).

From fig. 4, we can observe a fast convergence 
characteristics with a relatively small number of the multilayer thin-films layer and about 10 layers are enough to separate TE/TM mode. Since the fields have equal amplitudes in the two kind of layers for the TE polarization, while, the fields in the $\mathrm{S}_{i} \mathrm{O}_{2}$ layer is reduced by a factor of $\left(n_{H} / n_{L}\right)^{2}$ relative to the $\mathrm{T}_{\mathrm{i}} \mathrm{O}_{2}$ layer for the TM polarization, a strong polarization dependence is observed, and hence $\mathbf{n}_{\mathbf{T E}}>\mathbf{n}_{\mathbf{T M}}$. As a preliminary to the discussion, guided modes in the inner slab region support the TE1 , TE2 mode, and TM1 mode, while in the outer region depends on the layer number $\mathrm{N}$ (there are no guided mods in case of $\mathrm{N}=0$ ).

\section{$B$. Guided modes of rib-type 3-D waveguides}

A plot of the effective index of refraction as a function of line-width $(W)$ in the waveguide are shown in Fig.5 for the rib-type 3-D waveguide having thin-films layer number $\mathrm{N}=10$ in inner region, and $\mathrm{N}=0$ - 9 in outer regions. From the dispersion curve shown in Fig.5(a), we are observed that odd and even modes may cross but two odd and even modes never cross, because take place a strong mode coupling amang these modes. Likewise, Fig.5(c) shows the dispersion curve of the rib-type waveguide of multilayer thin-films $\mathrm{N}=10$ in the inner region and $\mathrm{N}=9$ in the outer regions. The waveguide operates as only a single-mode (TE-polarized mode in the range up to line-width $W=2.4 \mu \mathrm{m}$ ). Fig.5(d) also shows the contour plots of the field distribution corresponding to the points labeled on curves in Fig.5(a).

\section{Guided modes of strip-loaded type single and} parallel coupled line

Figs.6 (a),(b) show the dispersion curves of striploaded type single and parallel waveguide on the multilayer thin-films. When the thickness of strip is $0.3 \mu \mathrm{m}, 3-\mathrm{D}$ optical waveguide operates as a single mode and polarization-maintaining waveguide up to line-width $4 \mu \mathrm{m}$.

From these results, following conclusions are also obtained for the rib and strip loaded-type 3-D waveguide with multilayer thin-films of $\mathrm{SiO}_{2}$ and TiO2 materials.

\section{About $\mathrm{N}=10$ layers are enough to separate $\mathrm{TE}$ and} TM mode sufficiently.

2. Ratio of $\mathrm{dH} / \mathrm{dL}=0.2$ gives the reasonable effective refractive index for the dominant mode.

3. Number of thin-film layer at the rib-type side structure can surely control the unwanted higher modes to radiate.

4. $25 \mathrm{TE}$ and $25 \mathrm{TM}$ modes are roughly enough to describe the field distributions of the 3-D optical waveguide.

\section{Conclusion}

A new single-mode and polarization-maintaining 3D rib-type waveguide having the multilayer thin-film structures are proposed,and its propagation properties of guided mode were analyzed by using the " modematching method" based on the planar circuit equations. When the propagation constant of the waveguide is needed to be calculated for a different guide width, this method does not need to calculate the equivalent circuit parameters again.

Furthermore,one can extend this method to calculate the eigenvalues for a directional coupler or mutiple coupled waveguides, based on this equivalent circuit model. The numerical results gave the complete set of hybrid modes included mode conversion effects. We also discussed on operating ranges of only a single-mode and TE polarized-mode in the three-dimensional optical waveguides with the multilayer thin-film structures. The proposed strip loaded-type multilayer thin-films waveguide is now being fabricated in our laboratory. Moreover, the multilayer thin-film structures considered herein will be useful as curved waveguide using asymmetric structure,parallel coupled waveguides and resonators not only in the optical frequency range,but also in the millimeter-wave and submillimeter-wave frequency ranges.

\section{Reference}

(1) M.Born and E. Wolf,Principles of Optics", MacMillan, New York,1964.

(2) J.P. van der Ziel, M. Ilegerns and R. M. Mikulyak, Optical birefringence of thin GaAs-AlAs multilayer films, Appl. Phys. lett., vol.28, No. 12, pp.735-737, 15 June, 1976.

(3) M. Kitagawa and M. Tateda, Form birefringence of periodic multilayers", Appl. Opt., vol. 24, No. 20, pp.3359. 3362, 15 Oct., 1985.

(4) M.R. Knox and P.P. Toulios, in Proc. Symp. Submillimeter Waves. New York: Polytechnic, 1970, p.497.

(5) P.C.Kendall, M.J. Adams, S. Ritchie, and M.J. Robertson, "Theory for calculationg approximate values for the propagation constants of an optical rib waveguide by weighting the refractive indices," IEE Proc., vol.134,1987

(6)B.M.A Rahman and J.B. Davies, "Vector-H finite element solutions of GaAs/GaAlAs rib waveguids," IEE Proc., vol.132,pp.349-353, 1985.

(7) S. T. Peng and A. A. Oliner, Guidance and Leakage properties of a class of open dielectric waveguides: Part I Mathematical formulations, IEEE Trans. MTT., vol. MTT29, pp. 843-854,1981.

(8) Hsu,Jui-Pang, T. Anada , F. Eriguchi, Proposal os surface-wave planar circuit, Formulation of its planar circuit equations and its practical application", 1986 IEEE MTT-S Digest., GG-4, pp.797 -800.

(9) Hsu,Jui-Pang, T. Anada, A. A. Oliner, S. T. Peng, Formulation of mode coupling equations at step discontinuity based on the planar circuit theory", 1989 IEEE MTT-S Digest. , pp-5, pp.1135- 1138. 
Table 1 Fundamental relation of surface-wave planar circuit

\begin{tabular}{|c|c|c|c|}
\hline & TE (H) MODE & TM (E) MODE & \\
\hline 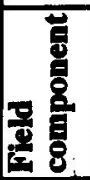 & $\begin{array}{l}H_{z}(x, y, z) \equiv-V_{n}^{H}(x, y) \cdot g_{n}^{H}(z) \\
E_{t}(x, y, z) \equiv\left[J_{n}^{H}(x, y) \times \mathbf{k}\right] \cdot f_{n}^{H}(z) \\
H_{t}(x, y, z) \equiv\left(m_{0}\right)^{-1} J_{n}^{H}(x, y) \cdot h_{n}^{H}(z)\end{array}$ & $\begin{aligned} E_{z}(x, y, z) & =-V_{n}^{E}(x, y) g_{n}^{E}(z) \\
E_{t}(x, y, z) & \equiv\left[k^{\prime} \dot{x} J_{n}^{E}(x, y)\right] f_{n}^{E}(z) \\
H_{t}(x, y, z) & \equiv j \eta_{0} J_{n}^{E}(x, y), h_{n}^{E}(z)\end{aligned}$ & $\begin{array}{l}\text { A } \\
\text { B } \\
\text { C }\end{array}$ \\
\hline 列 & $\begin{array}{ll}\operatorname{gradV}=-Z_{n}^{H} J_{n}^{H}, \quad Z_{n}^{H}=j \frac{\left(\beta_{n}^{H}\right)^{2}}{\omega \mu}(S / m) \\
\operatorname{div} J_{n}^{H}=-Y_{n}^{H V}, \quad Y_{n}^{H}=j \omega \mu \quad(\Omega / m) \\
\beta_{t}=\beta_{n}^{H}, Z_{n n}^{H}=\frac{\beta_{n}^{H}}{\omega \mu}(S), Y_{o n}^{H}=\frac{\omega \mu}{\beta_{n}^{H}}(\Omega)\end{array}$ & 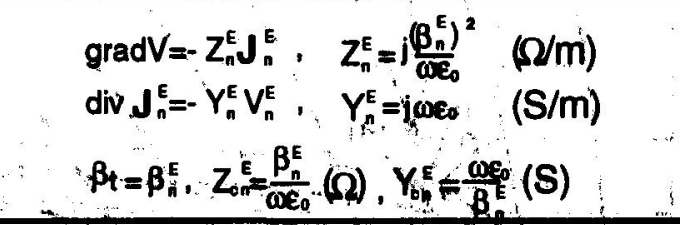 & $\begin{array}{l}\mathbf{D} \\
\mathbf{E} \\
\mathbf{F}\end{array}$ \\
\hline 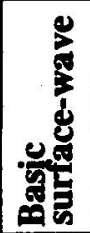 & $\begin{array}{l}\frac{d^{2} g^{H}}{d z^{z}}+\left[k_{0}^{2} \varepsilon_{s}(z)-\left(\beta_{n}^{H}\right)^{2}\right] g_{n}^{H}=0 \\
f_{n}^{H}=g_{n}^{H}, h_{n}^{H}=\frac{1}{k_{0}} \frac{d f_{n}^{H}}{d z} \\
\left\langle g_{m}^{H}, f_{n}^{H}\right\rangle=\int g_{m}^{H} f_{n}^{H} d z=\delta_{m}\end{array}$ & 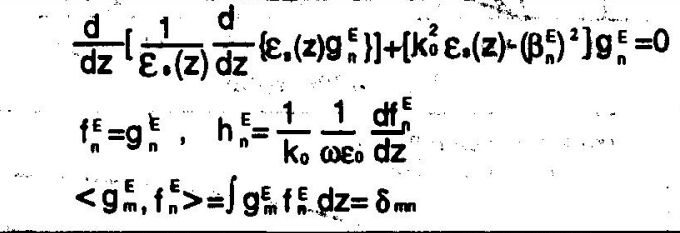 & $\mathbf{G}$ \\
\hline
\end{tabular}

$\mathbf{k}$ :unit vector toward height direction

$k_{0}=\omega_{0} \sqrt{\varepsilon_{0} \mu} \quad \eta_{0}=\sqrt{\mu \varepsilon_{0}}$

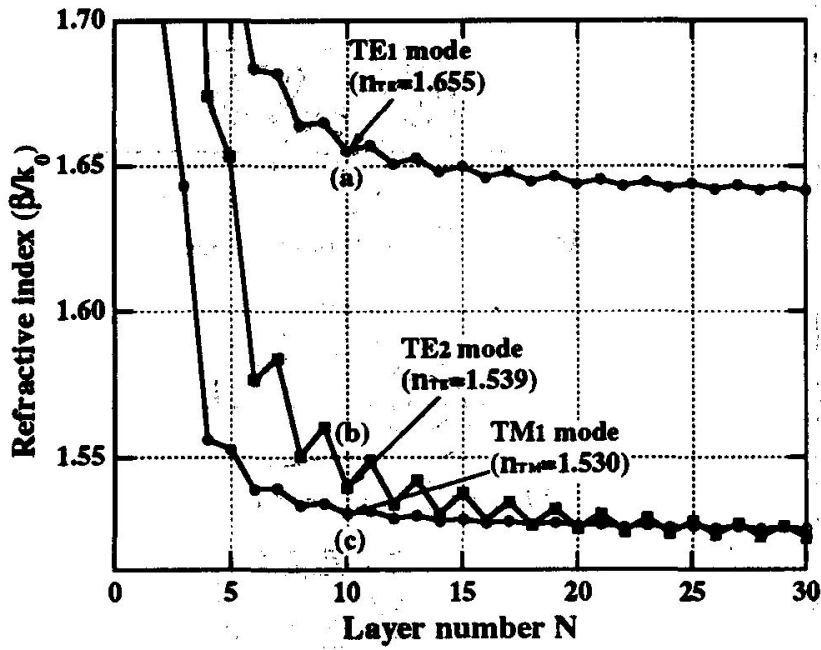

Fig.4(a) Effective refractive index of slab waveguide as a function of thin-film layer number $\mathbf{N}$.

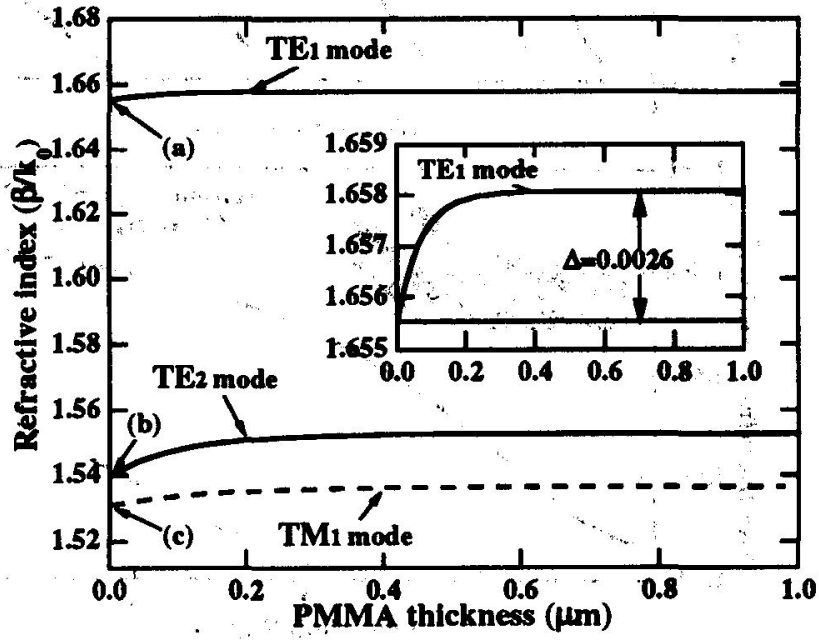

Fig,4(b) Modal refractive index as a function of PMMA thicliness ( $d_{p}$ ) loaded on multilayer thin-films slab.
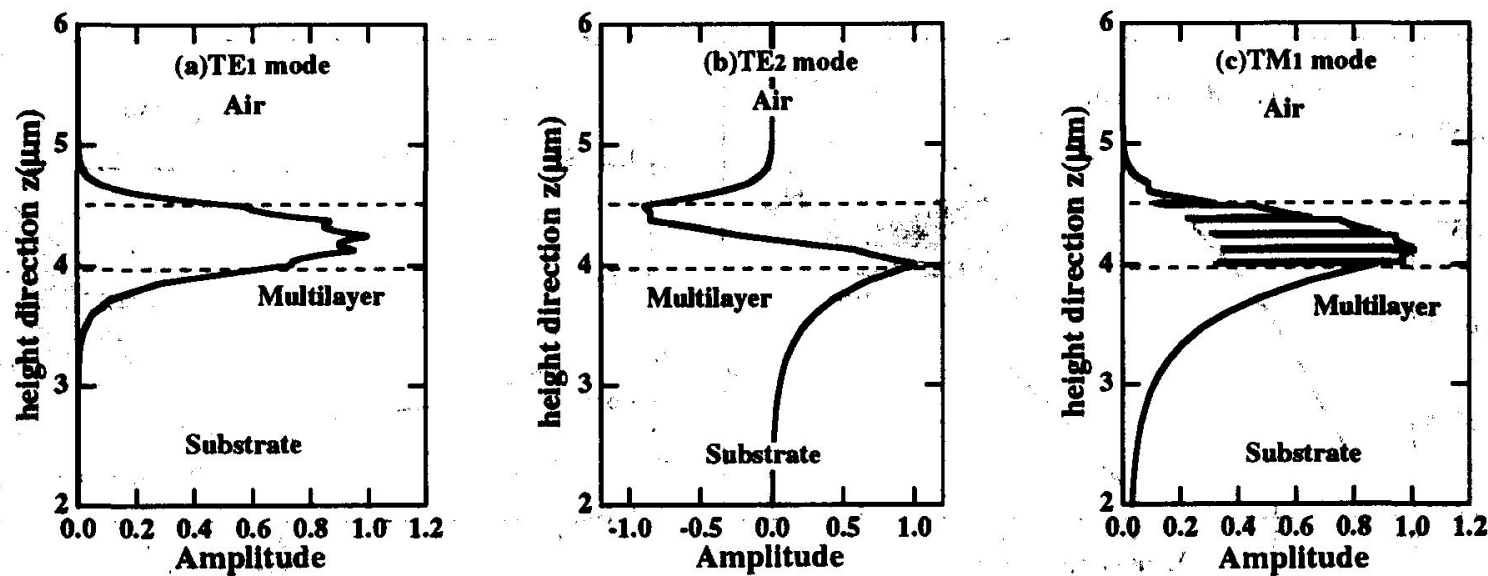

Fig.4(c) Field distributions of multilayer thin-film slab waveguide:

( (a): TE1 mode (b): TE2 mode (c): TM1 mode) 

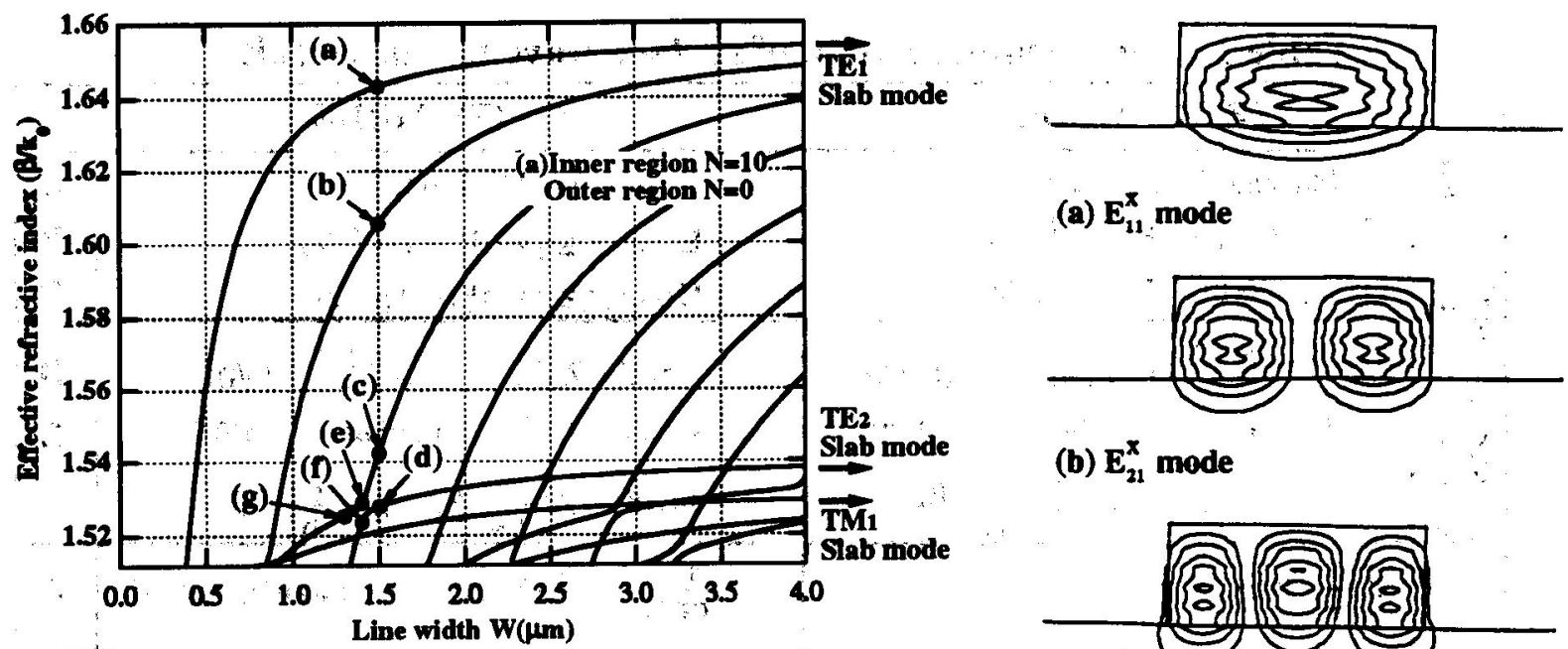

(a) $E_{11}^{x}$ mode

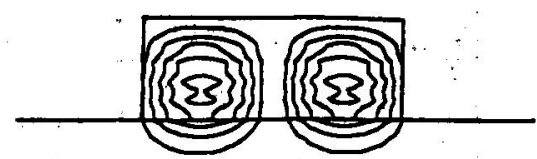

(b) $E_{21}^{x}$ mode

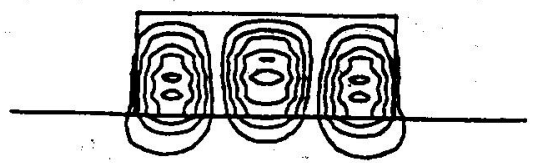

(c) $E_{31}^{x}$ mode
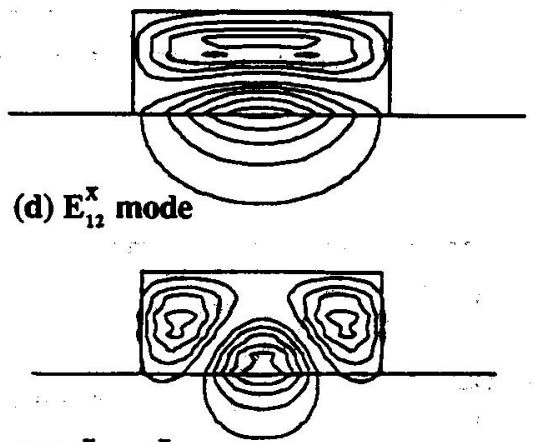

(e) $E_{31}^{x}+E_{12}^{x}$ combined mode
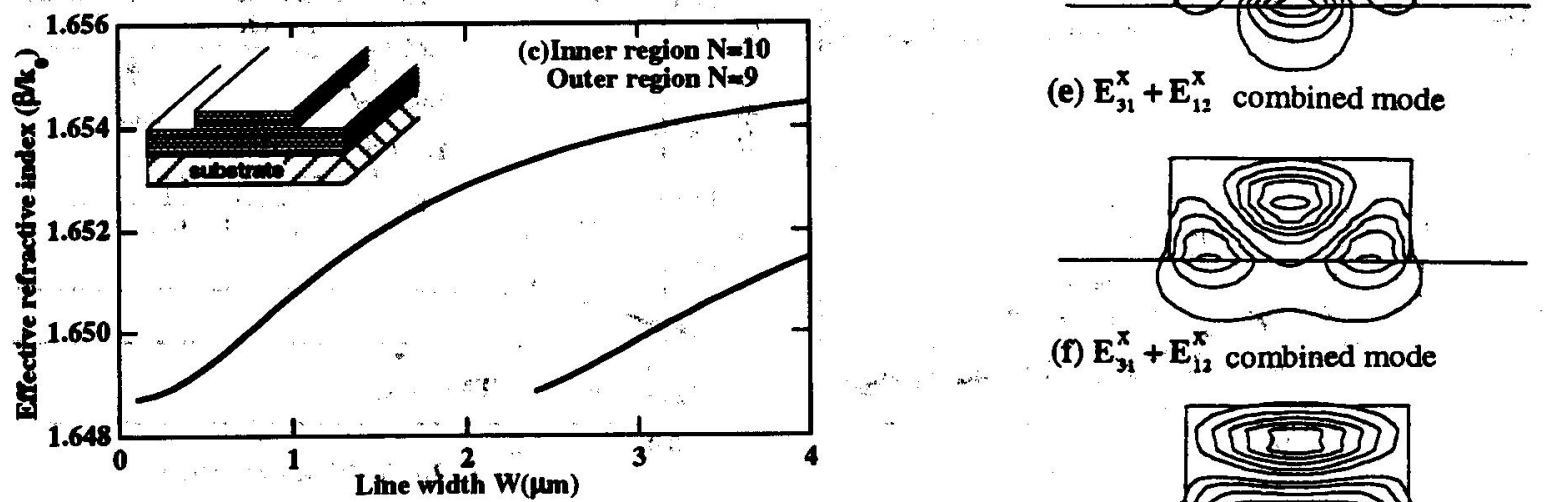

(f) $E_{31}^{x}+E_{12}^{x}$ combined mode

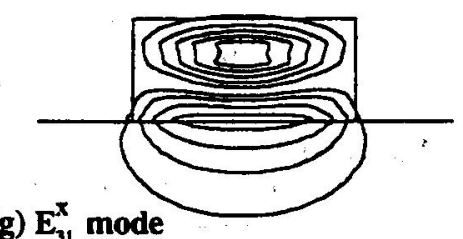

Fig.5 Modal refractive index as a function of He-width for multilayer thin-film steptype 3-D waveguide and field distributions.

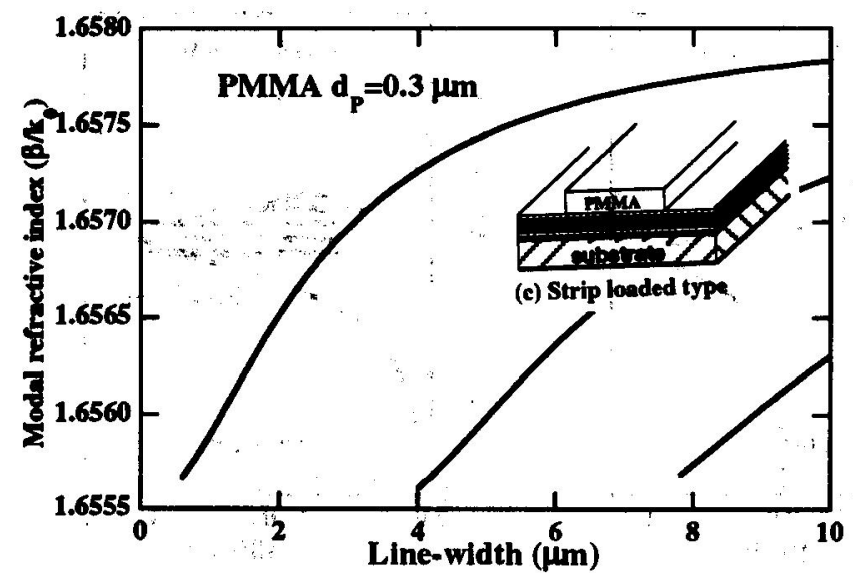

Fig.6(a) Modal refractive index ploted as a function of line-width in strip loaded-type 3-D waveguide.

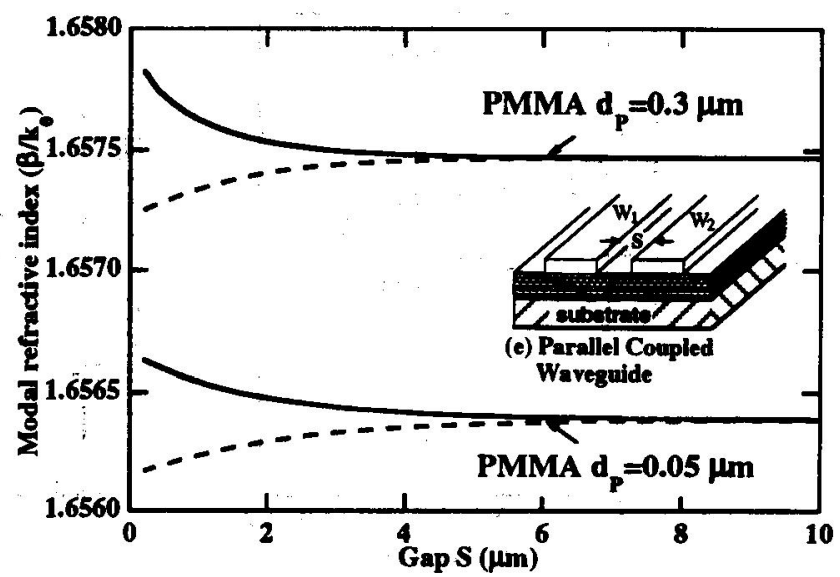

Fig.6(b) Modal refractive index of even and odd modes as a function of the guide separation $S$ for paralle coupled line. 